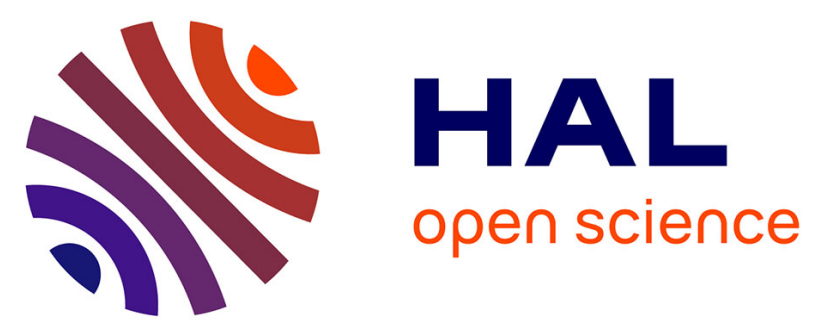

\title{
Use of OFDM-based digital TV for ranging: tests and validation on real signals
}

\author{
Damien Serant, Lionel Ries, Paul Thevenon, Olivier Julien, Christophe \\ Macabiau, Mathieu Dervin, Marie-Laure Boucheret
}

\section{- To cite this version:}

Damien Serant, Lionel Ries, Paul Thevenon, Olivier Julien, Christophe Macabiau, et al.. Use of OFDM-based digital TV for ranging: tests and validation on real signals. NAVITEC 2010, 5th ESA Workshop on Satellite Navigation Technologies and European Workshop on GNSS Signals, Dec 2010, Noordwijk, Netherlands. pp 1-8, 10.1109/NAVITEC.2010.5708017 . hal-01022202

\section{HAL Id: hal-01022202 \\ https://hal-enac.archives-ouvertes.fr/hal-01022202}

Submitted on 29 Sep 2014

HAL is a multi-disciplinary open access archive for the deposit and dissemination of scientific research documents, whether they are published or not. The documents may come from teaching and research institutions in France or abroad, or from public or private research centers.
L'archive ouverte pluridisciplinaire HAL, est destinée au dépôt et à la diffusion de documents scientifiques de niveau recherche, publiés ou non, émanant des établissements d'enseignement et de recherche français ou étrangers, des laboratoires publics ou privés. 


\title{
Use of OFDM-based Digital TV for Ranging: Tests and Validation on Real Signals
}

\author{
Damien Serant \\ Ecole Nationale de l'Aviation Civile/TéSA \\ Toulouse, France \\ serant@recherche.enac.fr
}

\author{
Lionel Ries, Paul Thevenon \\ Centre National d'Etudes Spatiales \\ Toulouse, France
}

\author{
Olivier Julien, Christophe Macabiau \\ Ecole Nationale de l'Aviation Civile \\ Toulouse, France
}

\author{
Marie-Laure Boucheret \\ University of Toulouse, IRIT/ENSEEIHT/TéSA \\ Toulouse, France
}

\begin{abstract}
This article presents results of tests on real signal of a method of positioning using a digital TV DVB-T signal-ofopportunity which is based on an OFDM modulation. OFDM and DVB-T standard are presented and the positioning method using the DVB-T signal is detailed. The result on real signal are analyzed qualitatively and quantitatively in static and dynamic scenarios and indoor and outdoor environments. In static scenarios, the standard deviation of pseudorange error is about $8 \mathrm{~cm}$ outdoor and $0.7 \mathrm{~m}$ indoor. In dynamic scenarios the pseudorange estimation is considerably disturbed and additional experiments are necessary to quantify the performance in this case.
\end{abstract}

Keywords- signal-of-opportunity, positioning, TV, OFDM, $D V B-T$

\section{INTRODUCTION}

Global Navigation Satellite System (GNSS) has grown significantly in recent years thanks to the development of many mass-market applications, such as car navigation, asset management or mobile positioning. However, in difficult environments such as dense urban or indoor areas, GNSS shows only limited performance in terms of accuracy and availability due to masking and/or reflections and/or diffraction of the direct signal. This compromises the use of GNSS as a standalone mean for robust positioning in challenging environments. Fortunately, urban areas have the advantage to have dense telecommunication networks to provide mobile phone, $\mathrm{TV}$, and all sorts of services to the user. The use of these signals is now strongly considered as a way to provide a rough estimation of the user position when GNSS is not available, or to assist GNSS in those environments. This is why these signals are often referred to as signals of opportunity. They include Wi-Fi, WiMAX, TV signals, mobile phone signals, etc... Several commercial positioning services based on signal of opportunity already exist : Rosum uses Time Difference of Arrival (TDOA) with
ATSC digital TV signals [1], Skyhook uses fingerprinting with Wi-Fi signals [2], etc...

The study presented in the paper targets the use of the European Digital Video Broadcast - Terrestrial (DVB-T) signals for positioning. This digital TV standard, although developed in Europe, has been widely accepted worldwide [3] and is the basis for other standards targeting handheld receivers, DVB-H (Handheld) and DVB-SH (Satelitte-toHandheld). The DVB-T signal uses an Orthogonal Frequency Division Multiplexing (OFDM) air interface. The advantages of TV signals for our purpose, especially when designed for handheld devices, are:

- the received signal power level is high enough to authorize an indoor or dense urban service area reception (which is often impossible with GNSS signals). Moreover, it is easy to understand that the typical received Signal to Noise Ratio (SNR) threshold for a communication service will likely be significantly higher than that required for ranging due to the necessity of demodulating the communication message with a low Bit Error Rate (BER). Therefore, as the broadcasting network has been designed to provide at least one emitter with power above the demodulation threshold, it will be likely that several emitters of this same network will be "available" for ranging.

- $\quad$ the wide bandwidth of TV signals (between 6 and 8 $\mathrm{MHz}$ ), larger than current mass-market GNSS signals, can potentially offer a better synchronization capability than the current GPS C/A.

- DVB-T signals (and all OFDM signals) contain pilot symbols that are used for equalization and can be used for synchronization. Those pilots can be used to construct a Delay Lock Loop (DLL) [4].

I would like to thank CNES and Thales Alenia Space France for funding this work 
- emitters are fixed which facilitates the position computation (emitter position monitoring is not required as in GNSS)

A ranging technique using DVB-T signals and its performances in an AWGN channel was introduced in earlier work [5]. This paper aims at assessing the proposed pseudorange estimation technique through experimental results using the French digital TV terrestrial network based on DVB-T standard.

This paper is organized as follow:

- The first section describes the principles of OFDM modulation and its related processing. This section also presents the DVB-T standard, including the signal parameters and the pilot structures.

- The second section deals with the software DVB-T receiver architecture used to process the recorded real signal, including the classical functions of a DVB-T receiver and the functions dedicated to positioning (i.e. delays acquisition and tracking).

- The third section gives a description of the test bench including the hardware devices used for the signal digitization and the software used to record the signal.

- The last section presents results of the tests on real signal including static and dynamic scenarios and indoor or outdoor environments.

\section{OFDM MODULATION AND DVB-T STANDARD}

\section{A. Orthogonal Frequency Division Multiplexing}

The concept of OFDM [6] consists in transmitting in parallel $N$ complex data symbols over $N$ orthogonal narrowband subcarriers (1 subcarrier carries 1 symbol). The width of these subcarriers is chosen narrow enough so that the channel frequency response can be considered as flat over the subcarrier bandwidth. The consequence is that channel equalization becomes very simple even in case of dense multipath environment. Thanks to the orthogonality of the subcarriers, their spectra can overlap without interfering with each other, allowing an excellent spectral efficiency and no Inter-Carrier Interference (ICI) when the receiver is synchronized.

An OFDM useful symbol is obtained by passing the $N$ complex data symbols through an inverse-Fast Fourier Transform (iFFT) operator. The useful part of the OFDM symbol is thus composed of $N$ samples. To take advantage of the FFT algorithm, $N$ is generally chosen to be a power of two. Conversely, the demodulation of an OFDM symbols is performed by a direct FFT.

Additionally, a guard interval of $N_{C P}$ samples is inserted before the useful OFDM symbols in order to avoid InterSymbol Interference (ISI). In the case of DVB-T, this guard interval, referred to as Cyclic Prefix (CP) is a replica of the last $N_{C P}$ samples of the OFDM symbol. By doing so, any demodulation of the OFDM symbol that is done with an Nsample FFT starting in the CP will only result in a phase rotation of each subcarrier proportional to its frequency offset, thus easily equalized. An OFDM symbol (useful symbol $+\mathrm{CP}$ ) is thus composed of $N+N_{C P}$ samples.

Fig. 1 illustrates an OFDM transmission/reception block diagram.

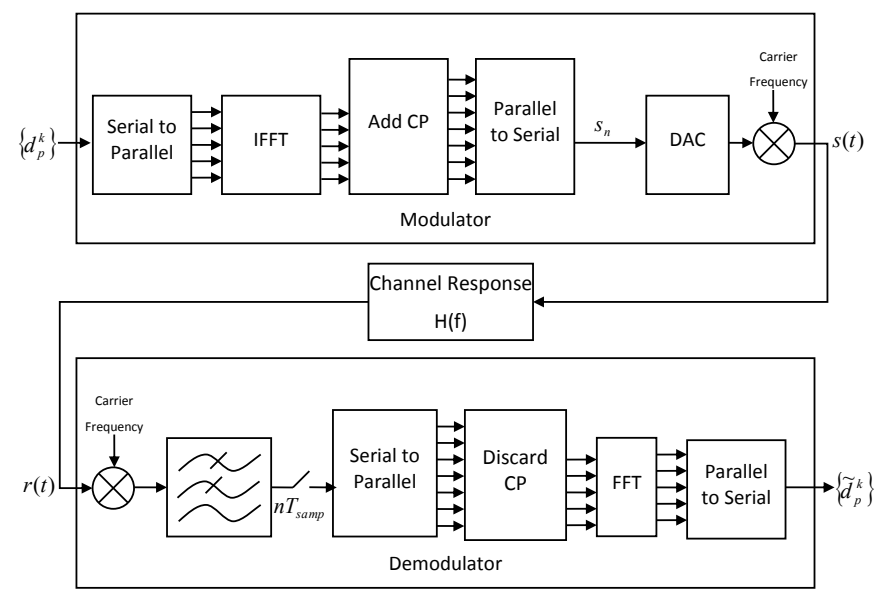

Figure 1. OFDM transmission/reception block diagram

According to Fig. 1, the expression of the n-th sample of the k-th emitted OFDM symbol, denoted $s_{n}^{k}$, is:

$$
\begin{gathered}
s_{n}^{k}=\frac{1}{N} \sum_{p=0}^{N-1} d_{p}^{k} \exp \left(j 2 \pi \frac{p n}{N}\right)=i F F T\left(d_{p}^{k}\right)[n] \\
\text { with }-N_{C P} \leq n \leq N-1
\end{gathered}
$$

where:

- $n$ is the sample time index,

- $\quad p$ is the subcarrier index,

- $\quad k$ is the OFDM symbol number,

- $d_{p}^{k}$ is the $\mathrm{p}$-th complex data symbol of the $\mathrm{k}$-th OFDM symbol and

- $\quad s_{n}^{k}$ is the n-th sample of the k-th OFDM symbol.

The sampled received signal is affected by several impairments due to the propagation channel and imperfections of the emitter and receiver:

- Carrier Frequency Offset (CFO), noted $\Delta f$ that occurs when the receiver local oscillator (LO) used for down conversion does not match with the carrier of the received signal. It results in two factors: frequency mismatch between emitter and receiver LO, and the resulting Doppler effect due to the movement of the receiver and/or the emitter. It is usually normalized by the frequency separation between 2 subcarriers $\left(1 / T_{U}\right) . T_{U}$ is also the duration of the useful part of an OFDM symbol ( $N$ samples). This CFO can be decomposed into an integer and a fractional part so that $\Delta f=\delta f+p_{f}$, with $\delta f \in$ $\left[-0.5,0.5\left[\right.\right.$ and $p_{f} \in \mathbb{N}$. 
- Sampling Clock Offset (SCO), denoted $\delta_{S C O}$, is due to the sampling clock frequency mismatch between emitter and receiver and the Doppler effect. Its value is expressed in part per million (ppm), which corresponds to a clock drift of $1 \mu$ s per second.

- Timing Offset, denoted $\Delta \tau$, corresponding to the time difference between the estimated and the real beginning of one OFDM symbol normalized by the sampling period. This means that the FFT made by the receiver is not perfectly synchronized with the OFDM useful symbol.

- $\quad$ Distortion of the channel with a transfer function $H$ and an impulse response $h$.

- $\quad$ Additive White Gaussian Noise (AWGN) $n(t)$.

Thus the received signal is:

$$
r(t)=(s(t) * h(t))\left(t-\Delta \tau . T_{\text {samp }}\right) e^{j 2 \pi \frac{\Delta f}{T_{U}} t}+n(t)
$$

This received signal is sampled at the sampling period $\left(1+\delta_{S C O}\right) \cdot T_{\text {samp }}$, where $T_{\text {samp }}$ denotes the required sampling period, and grouped by $N+N_{C P}$ samples to form the vectors $\left\{r_{p}^{k}\right\}_{p \in\left[-N_{C P} N-1\right], k \in \mathbb{N}}$

Taking into account all these impairments and assuming that the FFT windows taken by the receiver begin within the $\mathrm{CP}$ and after the end of the last replica of the previous OFDM symbol (i.e. no Inter-Symbol-Interference), the p-th demodulated subcarrier of the k-th received OFDM symbol is [7]:

$$
\begin{aligned}
\widetilde{d_{p}^{k}}= & F F T\left(\left(r_{n}^{k}\right)_{n=0}^{N-1}\right)[p] \\
= & d_{\tilde{p}}^{k} \cdot \alpha\left(\delta f+\delta_{S C O} \tilde{p}\right) \cdot H_{\tilde{p}}^{k} \cdot \exp \left(j 2 \pi \frac{\Delta \tau \tilde{p}}{N}\right) \times \\
& \exp \left(j \phi_{k}\left(\Delta f+\delta_{S C O} \tilde{p}\right)\right)+I C I+n_{p}^{k}
\end{aligned}
$$

where:

- $\quad \tilde{p}=\left(p+p_{f}\right) \bmod N$ the subcarrier index circularly rotated as a result of the integer CFO.

- $\widetilde{d_{p}^{k}}$ is the demodulated symbol over the p-th subcarrier of the k-th OFDM symbol,

- $n_{p}^{k}$ is the noise on the $\mathrm{p}$-th subcarrier of the $\mathrm{k}$-th OFDM symbol,

- $\quad H_{p}^{k}$ is the channel frequency response over the $\mathrm{p}$-th subcarrier of the k-th OFDM symbol,

- $\alpha(x)=\frac{\sin c(\pi x)}{\sin c\left(\frac{\pi x}{N}\right)} \exp \left(\pi x \frac{N-1}{N}\right)$ is the complex attenuation due to $\mathrm{SCO}$ and $\mathrm{CFO}$,

- $\phi_{k}(x)=2 \pi \frac{\left(k\left(N+N_{C P}\right)+N_{C P}\right)}{N} x$ is the phase rotation due to the SCO and CFO and depending on the OFDM symbol number $k$, and

- ICI the inter-carrier interference
According to (3) a demodulated symbol is equal to the transmitted one if the integer part of the $\mathrm{CFO}$ is equal to zero (if not, a different transmitted symbol is demodulated instead), and if it is correctly phase-compensated by some complex factors dependent on the different aforementioned impairments. The integer CFO needs to be estimated and corrected in order to recover the transmitted symbols in the right order. The complex factor can be easily corrected by the channel equalization module. However fractional CFO and SCO also induce ICI which can be considered as noise. Consequently those errors need to be corrected before demodulation in order to limit the noise on the demodulated symbols. In AWGN channel and for a high SNR, the degradation of SNR due to ICI (rising from the uncompensated $\mathrm{CFO}$ and $\mathrm{SCO})$ is [8][9]:

$$
D_{I C I}^{d B}(p) \approx 10 \cdot \log _{10}\left(1+\frac{\pi^{2}}{3} S N R \cdot\left(\delta f+\delta_{S C O} p\right)^{2}\right)
$$

The fractional CFO may often reach its maximum value of 0.5 and then the SNR degradation can be several tens of $\mathrm{dB}$. Thus the estimation and the correction of the fractional CFO need to be done before the demodulation in order to limit the induced ICI. The classical values of the SCO (about $10 \mathrm{ppm}$ ) induces a limited SNR degradation (a few $\mathrm{dB}$ ). Thus, the estimation of the SCO can be done after demodulation. Moreover for small values of SCO (less than about $5 \mathrm{ppm}$ ) the correction is not necessary since the SNR degradation is negligible.

\section{B. Digital Video Broadcasting - Terrestrial}

The DVB-T [10] is a European standard based on an OFDM air-interface for digital TV broadcasting to fixed receivers in the VHF and UHF bands. DVB-H (Handheld) and DVB-SH (Satellite-to-Handheld), both based on DVB-T, target mobile $\mathrm{TV}$ receivers and other potential frequency bands. These standards define a family of OFDM signals which depends on three parameters: the FFT size $(N)$, the ratio between the Cyclic Prefix length and the useful OFDM symbol length ( $\left.C P=N_{C P} / N\right)$ and the sampling period $\left(T_{\text {samp }}\right)$. Table I shows different possible values defined in the DVB-T standard for each parameters.

TABLE I. DVB-T PARAMETERS

\begin{tabular}{|c|l|}
\hline Parameters & \multicolumn{1}{|c|}{ Possible values } \\
\hline $\boldsymbol{N}$ & $2048($ Mode $2 \mathrm{~K}), 4096$ (Mode 4K) and 8192 (Mode 8K) \\
\hline $\boldsymbol{C P}$ & $1 / 32,1 / 16,1 / 8,1 / 4$ \\
\hline $\boldsymbol{T}_{\text {samp }}$ & $\begin{array}{l}7 / 64 \mu \mathrm{s}(8 \mathrm{MHz} \text { channels), } 1 / 8 \mu \mathrm{s}(7 \mathrm{MHz}), 7 / 48 \mu \mathrm{s}(6 \\
\mathrm{MHz} \text { ) and } 7 / 40 \mu \mathrm{s}(5 \mathrm{MHz})\end{array}$ \\
\hline
\end{tabular}

The $N$ subcarriers of the useful OFDM symbol have different natures:

- Null subcarriers which are located on the edges of the signal spectrum and have a zero value. They serve as guard bands to avoid out-of-band emissions of the OFDM signal. 
- Data subcarriers which are QAM-modulated and have their amplitude normalized in such a way that their variance is unitary.

- Transmission Parameter Signaling (TPS) subcarriers which are BPSK-modulated and carry information about the actual transmission parameters.

- Pilot subcarriers which are BPSK-modulated. Their value is given by a known Pseudo-Random Binary Sequence (PRBS). Their amplitude is boosted by a factor of $4 / 3$ compared to Data and TPS subcarriers. Pilot subcarriers are used for several synchronization functions and for channel estimation.

The pilot subcarriers can be of two types: continuous pilots and scattered pilots. Continuous pilots are always located on the same subcarriers on every OFDM symbols. On the contrary scattered pilots are inserted every 12 subcarriers and the first pilot subcarrier index takes four different values $(3,6,9$ or 12) depending on the OFDM symbols number. Therefore the scattered pilot pattern repeats every 4 OFDM symbols. This organization is illustrated in Fig. 2 where only non-null subcarriers are represented.

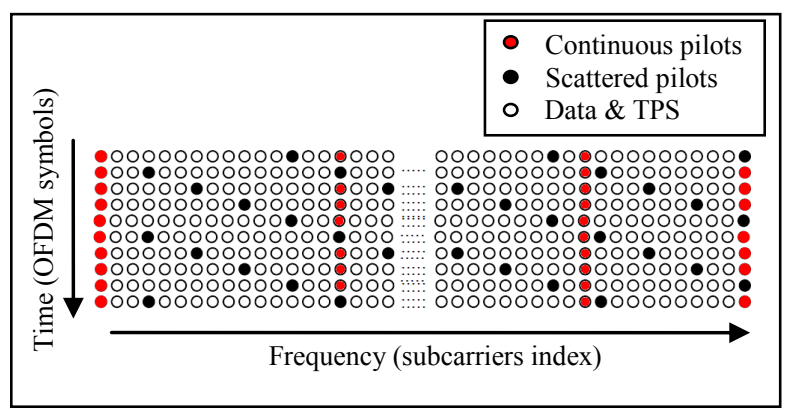

Figure 2. DVB-T pilot organization

\section{DESCRIPTION OF THE DVB-T SOFTWARE RECEIVER}

The developed DVB-T software receiver can be decomposed in two parts : (1) a communication part that gather classical functions of a DVB-T receiver such as timing and frequency synchronization or pilot sequence detection and (2) a navigation part that computes pseudoranges.

\section{A. Decription of the Communication Part}

The communication part of the developed DVB-T software receiver is composed of 3 main functions:

- Timing and Frequency synchronization: based on the Van De Beek algorithm [11], this function gives an estimation of the timing offset $\Delta \tau$ and of the fractional part of the frequency offset $\delta f$.

- Estimation of the integer part of frequency offset [7].

- Estimation of the scattered pilot sequence related to the OFDM symbol number [7].

\section{B. Description of the Navigation Part}

The navigation part is composed of 2 main functions: delay acquisition and delay tracking. Both of them require the computation of a correlation function. The terrestrial channel induces the presence of several strong multipaths in the received signal. The developed tracking strategy, introduced in [12], consists in trying to acquire and track a large number of multipaths in order to keep only the shortest one that is hoped to be the direct path. Tracking several signals also allows to go through some strong fading affecting certain signals. In addition it is necessary to periodically restart the delay acquisition in order to be able to track appearing signals potentially closer to the direct path.

\section{1) The correlation function}

The correlation function is computed by correlating the received signal with a local replica generated only with the pilot subcarriers. Fig. 3 illustrates this principle.

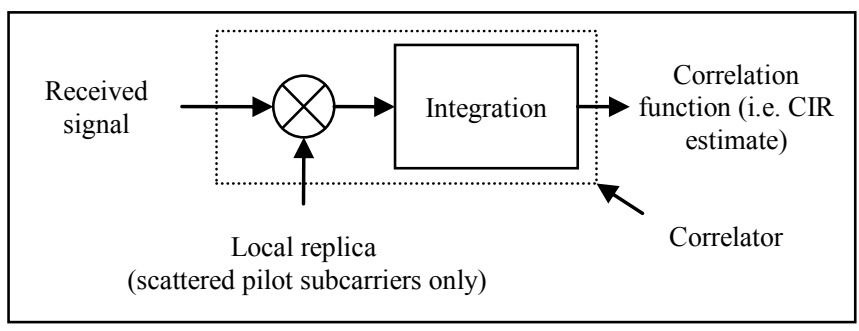

Figure 3. Correlation principle

To take advantage of the efficient FFT used for OFDM demodulation, the correlation function computation involves the demodulated symbols and the pilot values, as expressed in (5).

$$
R^{k}(\tau)=\frac{1}{N_{p}} \sum_{p \in \mathcal{P}_{S}(k)} \widetilde{d_{p}^{k}} P_{p}^{k^{*}} \exp \left(j 2 \pi \frac{\tau p}{N}\right)
$$

Where:

- $\tau$ is the correlation delay normalized by the sampling period,

- $\quad R^{k}(\tau)$ is the value of the correlation function at $\tau$ for the k-th OFDM symbol,

- $\widetilde{d_{p}^{k}}$ is the demodulated symbol over the p-th subcarrier of the k-th OFDM symbol,

- $\quad P_{p}^{k}$ is the value $(-1$ or +1$)$ of the pilot over the $\mathrm{p}$-th subcarrier of the k-th OFDM symbol,

- $\mathcal{P}_{S}(k)$ is the set of scattered pilot indexes for the k-th OFDM symbol,

- $\quad N_{p}$ the number of scattered pilot subcarriers.

As demonstrated in [5], in an AWGN channel with an attenuation $\alpha$, the resulting absolute value of the correlation function for small delay values expressed in (6), is plotted on Fig. 4.

$$
|R(\tau)|=\frac{4}{3} \alpha \cdot\left|\operatorname{sinc}\left(\pi \beta \varepsilon_{\tau}\right)\right|
$$


where:

- $\varepsilon_{\tau}=\Delta \tau-\tau$

- $\operatorname{sinc}(x)=\sin (x) / x$

- $\beta=\frac{12 N_{p}}{N}$

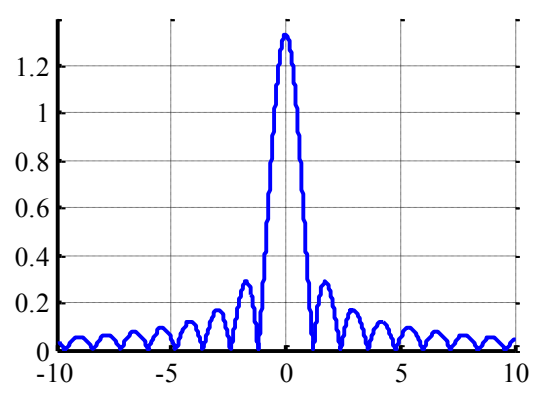

Correlation delay (relative to the sample period)

Figure 4. Absolute value of correlation function

\section{Coherent and non-coherent summations}

In order to reduce noise effect it is possible to use coherent and non-coherent summations of the correlator outputs. Let $N_{c}$ be the number of coherent summations and $N_{I}$ the number of non-coherent summation. Then averaged correlation is expressed as:

$$
R_{N_{C}, N_{I}}(\tau)=\frac{1}{N_{I}} \sum_{l=0}^{N_{I}-1}\left|\frac{1}{N_{C}} \sum_{k=0}^{N_{C}-1} R^{k+l N_{C}}(\tau)\right|^{2}
$$

\section{Windowed Correlation}

The goal of windowed correlation is to limit the amplitude of secondary lobes of the classical correlation and thus reduces their role in the well-known near-far problem [12]. The expression of the windowed correlation, denoted $R_{w}^{k}$ is presented on (8).

$$
R_{w}^{k}(\tau)=\frac{1}{N_{p}} \sum_{p \in \mathcal{P}_{S}(k)} \widetilde{d_{p}^{k}} P_{p}^{k^{*}} \exp \left(j 2 \pi \frac{\tau p}{N}\right) w(p)
$$

where:

- $w(p)=1$ for the rectangular window (i.e. no windowing),

- $w(p)=0.53836-0.46164 \cdot \cos \left(2 \pi \frac{p}{K-1}\right) \quad$ for hamming window,

- $\quad w(p)=0.35875-0.48829 \cdot \cos \left(2 \pi \frac{p}{K-1}\right)+$ $0.14128 \cdot \cos \left(4 \pi \frac{p}{K-1}\right)-0.01168 \cdot \cos \left(6 \pi \frac{p}{K-1}\right)$ for the blackman-harris window,

- $\quad$ and $K$ is the number of non-null subcarriers in an OFDM symbol.

Fig. 5 compares the absolute values of the correlation function for those three windows.

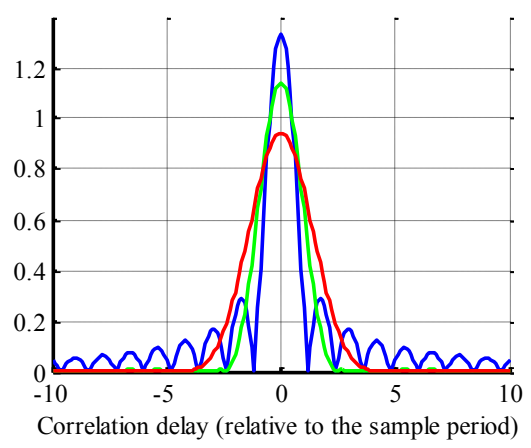

Figure 5. Absolute correlation function for Rectangular (blue), Hamming(green) and Blackman-Harris(red) windows

It can be seen that Hamming and Blackman-Harris windows reduce considerably the amplitude of secondary lobes in the correlation function. The counterpart is that the main lobe width is increased thus reducing the precision of the delay estimate.

\section{2) Delays Acquisition}

The correlation between the incoming signal and the local replica will be an estimation of the Channel Impulse Response (CIR). This CIR will be composed of a sum of delayed, attenuated and phase-modified windowed sinc function (plus noise and interference).

The first step towards the tracking of multiple paths is the acquisition of the delays of each individual path. This is done by identifying each peak of the estimated CIR, each peak corresponding to one delay of a multipath component of the received signal. As explained in details in [13], this is done by using the Matching Pursuit algorithm [14]. It consists in decomposing the absolute value of the estimated CIR in a sum of delayed and attenuated absolute reference functions (in the present case, one of the correlation functions plotted on Fig. 5 is the reference function) by iteratively (1) estimating the delay and amplitude of the highest peak of the remaining CIR and (2) subtracting the corresponding absolute sinc function (applying the estimated delay and amplitude) from the remaining CIR.

By using a threshold for the minimum desired amplitude of the signals of interest, or by setting a maximum number of signals to estimate, this decomposition provides an estimation of the delay and absolute amplitude of the main signal components of the received signal. The drawback of this technique is that it uses the absolute value of the correlation function and is cumbersome. However, it was showed to work sufficiently well for the present purpose.

\section{3) Delays Tracking}

All the delays acquired thanks to the Matching Pursuit algorithm are tracked using a classical delay lock loop (DLL). This DLL uses a normalized Early-minus-LatePower (EMLP) discriminator and a $2^{\text {nd }}$ order loop filter. Three criterions are defined to avoid to track uselessly a delay. A delay tracking is stopped when:

- the estimated SNR of the tracked peak passes under the tracking threshold (defined in [5]); 
- two tracked delay converge (then one of them is stopped);

- a delay estimate diverge unrealistically. [13].

More details on this DLL can be founded in [5] and

The overall tracking techniques was shown to work efficiently with simulated signals reproducing a realistic propagation channel [5][13]. The following sections will show the first tests realized using real signals.

\section{DESCRIPTION OF THE TEST BENCH}

The real signal acquisition is done thanks to two devices from Ettus LLC [15] called USRP2 and WBX. These devices are controlled by an open source software called GnuRadio [16].

USRP2, the motherboard, allows to digitize an analog baseband signal with a bandwidth up to $100 \mathrm{MHz}$ at a maximum sampling frequency of $100 \mathrm{MS} / \mathrm{s}$ (Mega-samples per second). The baseband signal is quantized on 14 bits on I\&Q channels. The digitized signal is sent to a PC through a gigabit Ethernet interface. Due to the limitation of Ethernet bandwidth, the actual maximum sampling frequency is 25 $\mathrm{MS} / \mathrm{s}$.

WBX, the daughterboard, plugged directly onto the USRP2, allows down-converting a received RF signal into an analog baseband signal which can be subsequently digitized by the USRP2. The WBX is capable to downconvert any kind of signal in the $50 \mathrm{MHz}-2.2 \mathrm{GHz}$ frequency range. An on-board adjustable amplifier from 0 $\mathrm{dB}$ to $31 \mathrm{~dB}$ can be used.

The goal of the experiment was to record a French digital TV signal, located in the $482 \mathrm{MHz}-826 \mathrm{Mhz}$ frequency range, which occupies a bandwidth of approximately $8 \mathrm{MHz}$. Consequently, the couple USRP2/WBX was used in reception and the down-converted signal is sampled at the sampling frequency of $100 / 12$ $\mathrm{MS} / \mathrm{S}$ (about $8.33 \mathrm{MS} / \mathrm{s}$ ).

A commercial omnidirectional TV antenna is connected to the WBX input to capture the TV signals. It is amplified with a gain of $20 \mathrm{~dB}$ and a noise factor of $3.5 \mathrm{~dB}$.

In addition, in order to have a stable sample frequency, the USRP2 is locked on a $10 \mathrm{MHz}$ external reference coming from a GPS receiver Novatel OEM4. Thus, the $10 \mathrm{MHz}$ is synchronized with the GPS time (with the GPS receiver precision). Fig. 6 summarizes this architecture.

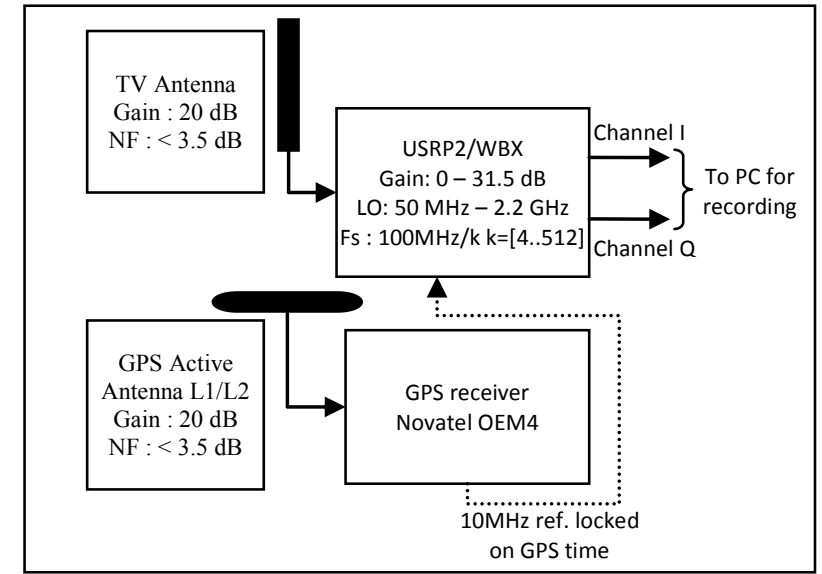

Figure 6. Architecture of the test bench

The URSP2 can be controlled by an open-source program called GNU Radio. GNU Radio is a software dedicated to the development of software-defined radio systems. It provides signal processing blocks, written in $\mathrm{C}++$ and the code structure to connect those blocks, in Python.

\section{Results Of Real Signal Tests}

Tests use the signal of an emitter located at about $80 \mathrm{~km}$ from the lab at an altitude of 1312 meters emitting at the central frequency of $554 \mathrm{MHz}$ with an EIRP of $5 \mathrm{~kW}$. A preliminary study showed that this particular emitter was synchronized to GPS time, which seems not to be the case of the other emitters of the region.

In the following are presented results of static outdoor, static indoor and dynamic indoor experiments. The correlation uses a rectangular windows and the loop bandwidth of the DLL is $1 \mathrm{~Hz}$.

\section{A. Static scenario}

For this first test, the antenna is placed on the roof of the lab at approximately 10 meters above the ground.

Fig. 7 shows the correlation image which represents the evolution of the CIR over the duration of the record. It is obtained by plotting, every 100 OFDM symbols, the correlation power over a large delay range between -3000 meters $(\sim-10 \mu \mathrm{s})$ and +6000 meters $(\sim 20 \mu \mathrm{s})$ around the rough delay estimate of the main signal path obtained by the Van-de-Beek algorithm (see section III). The color scale shows the correlation power in $\mathrm{dB}$ relative to the maximum power during all the simulation.

This figure shows two powerful peaks (about 20-25 dB above the noise floor) spaced by about 4000 meters corresponding to the direct path and a strong multipath. Secondary lobes of the sinc correlation function and/or close multipath clearly appear in light blue. 


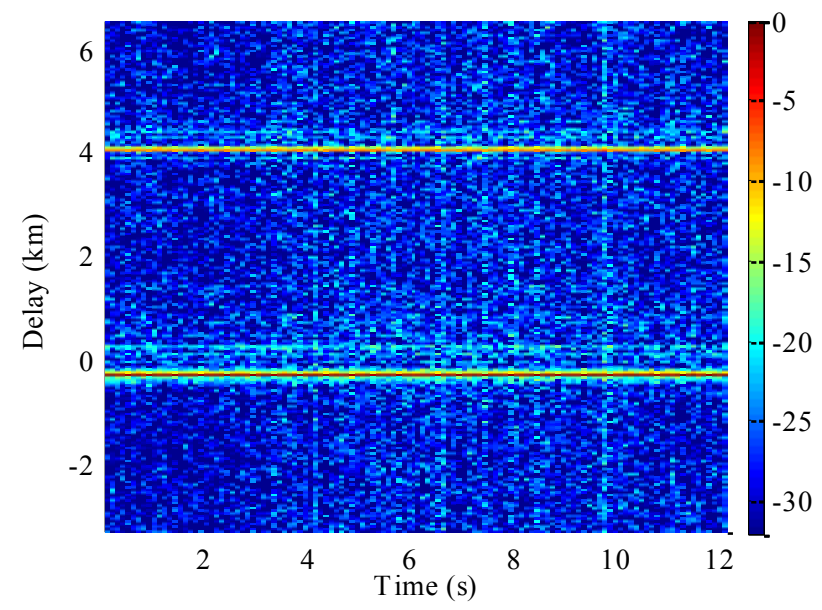

Figure 7. Correlation image - Static outdoor scenario

Fig. 8 represents the pseudorange error resulting from the tracking of the two powerful peaks of the correlation function (the blue curve corresponding to the shortest peak and the green to the other one).

The standard deviation of the pseudorange error (the real pseudorange variation being null since the emitter and receiver antennas are fixed) is about $8 \mathrm{~cm}$ for the two estimates. This is quite good even if the theoretical expression (presented in [5]) predicts a standard deviation of about $3 \mathrm{~cm}$. Since theoretical expression were obtained in an ideal case (no multipaths, no synchronization errors), it seems reasonable to have this difference. Because the two pseudorange errors are uncorrelated, they are not due to clock error (which should produced the same error), but more likely to noise and/or multipath effects.

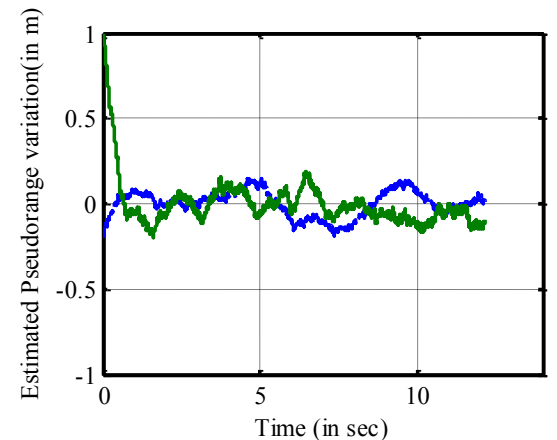

Figure 8. Pseudorange error - Static outdoor scenario

An SNR estimation is obtained thanks to the amplitude of the tracked peak. The estimated SNR for each tracked peak are about $-3.5 \mathrm{~dB}$ for the shortest one and $-9 \mathrm{~dB}$ for the other one.

In the second example, the antenna is located in a light indoor environment in the second floor of the building near a window.

The correlation image, plotted on Fig. 9, presents more short and strong multipaths and a higher noise floor than in the outdoor scenario. However the two strongest paths of the outdoor case are always presents.

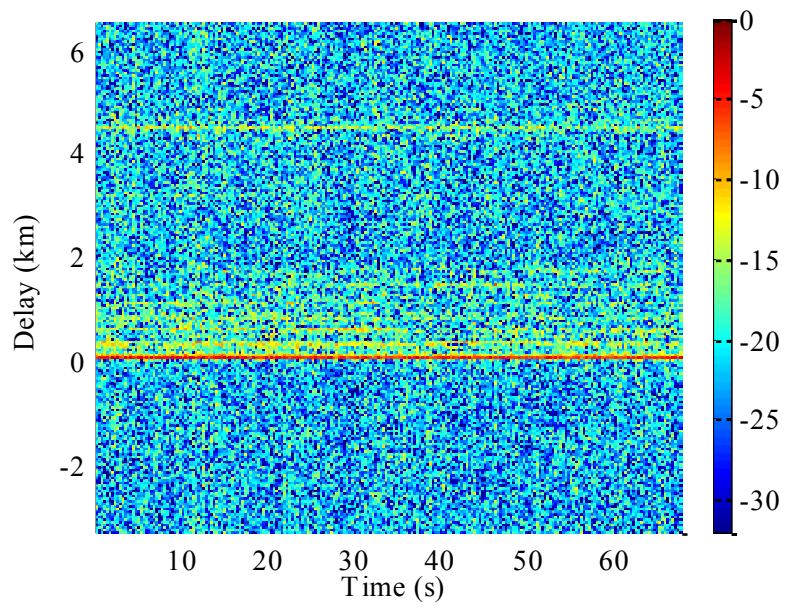

Figure 9. Correlation image - Static indoor scenario

Fig. 10 shows the pseudorange error corresponding to the tracking of the two same peaks as in the static outdoor scenario. The blue curve has a standard deviation of 0.7 meters and the green one of 2.3 meters. For information, at the estimated SNRs $(-13 \mathrm{~dB}$ for the first peak and $-25 \mathrm{~dB}$ for the second), the theoretical expression published in [5] gives a standard deviation of respectively 0.1 meters and 0.5 meters in an ideal case. The differences between theoretical and practical results are surely due to the omnipresence of strong multipaths in indoor environment.

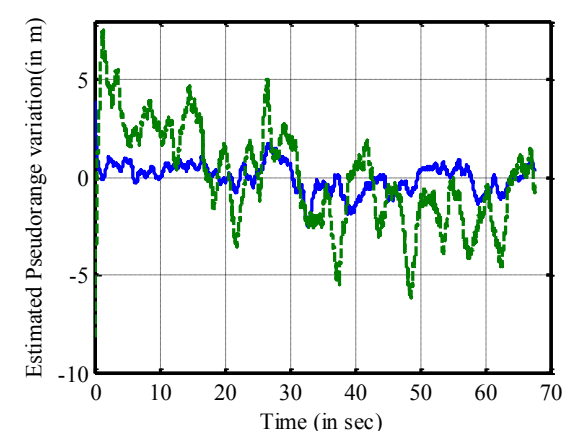

Figure 10. Pseudorange error - Static indoor scenario

\section{B. Dynamic Scenario}

In the following, dynamic indoor experiment the movement is divided into a succession of static and dynamic phases as follow : $15 \mathrm{~s}$ static, then $15 \mathrm{~s}$ dynamic, followed by $15 \mathrm{~s}$ static, then $12 \mathrm{~s}$ dynamic and finally $15 \mathrm{~s}$ static. The beginning of the test was near a window, while the rest of the test can be considered as deep indoor.

Fig. 11 illustrates the correlation image obtained in this experiment. Static and dynamic phase are easily recognizable: in the static phases the correlation is stable whereas in the dynamic phases correlation is highly disturbed. Here the fast fading phenomenon well known of urban and indoor channels is clearly observable.

This observation is also very clear when observing the estimated SNR on Fig. 12. 


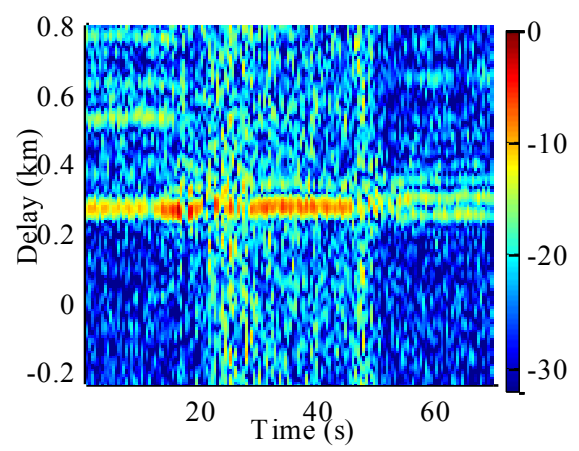

Figure 11. Correlation image - Dynamic indoor scenario

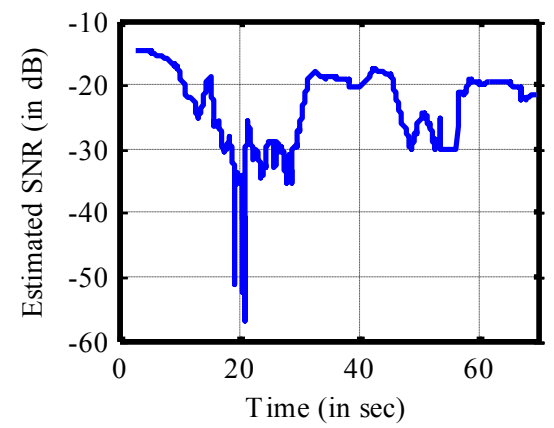

Figure 12. Etimated SNR - Dynamic indoor scenario

Fig. 13 shows the variation of the estimated pseudorange. In this case, this plot does not represent the pseudorange error since no reference position was available and receiver was moving. However it can be seen that during the dynamic phase the estimated pseudorange is strongly disturbed and that a stabilization appears during static phases. This can be understood when looking at Fig. 13 that shows a clear drop in the estimated SNR values during dynamic phases.

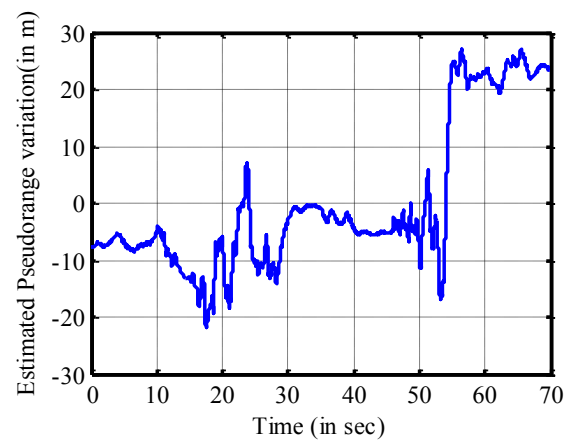

Figure 13. Pseudorange variation - Dynamic outdoor scenario

Unfortunately, because of real test constraints, a reference position was unavailable and the covered distance was limited. As a consequence a quantitative performance of the technique in a dynamic scenario can't be provided but it is clear that dynamic induces an important degradation of pseudorange estimation performance compared to static case.

\section{CONCLUSIONS AND FUTURE WORK}

A pseudorange computation method using digital-TV signal based on DVB-T standard, using OFDM modulation, has been introduced.
Tests of this method on real signal from a French digital TV emitter have been presented. Static scenarios present a small pseudorange error $(<1$ meter $)$, even in indoor environment. Those results are fairly close to theoretical predictions [5] although slightly degraded by the presence of numerous and strong multipaths. In dynamic scenarios the pseudorange error is strongly increased, especially because of fast fading phenomenon typical of urban and indoor propagation channels. Unfortunately the lack of measurements in this scenario does not permit to give a quantitative conclusion.

Future work will consist in a better characterization of the pseudorange error in dynamic scenarios by using a more complex test setup using a reference position (thanks to GPS for example) and increasing the travelled distance during the experiment (the real pseudorange variation has to be an order larger than the typical pseudorange variation due to fast fading to be representative). For this purpose a mobile and autonomous test bench is being developed.

\section{REFERENCES}

[1] "Rosum FemtoSynch In-Building Timing and Location for Femtocells",

www.rosum.com/Rosum_FemtoSynch_White_Paper_Oct08.pdf. Rosum Corporation, $200 \overline{8}$.

[2] “SKYHOOK Wireless.", www.skyhookwireless.com/howitworks/

[3] "DVB - Digital Video Broadcasting - DVB Worldwide." http://www.dvb.org/about_dvb/dvb_worldwide/

[4] E. Kaplan et C. Hegarty, Understanding GPS: Principles And Applications, Artech House, 2005

[5] D. Serant, P. Thevenon, M.-L. Boucheret, O. Julien, C. Macabiau, S. Corazza, M. Dervin, L. Ries, "Development and validation of an OFDM/DVB-T sensor for positioning," Position Location and Navigation Symposium (PLANS), 2010 IEEE/ION , vol., no., pp.9881001, 4-6 May 2010

[6] J.A. Bingham, "Multicarrier modulation for data transmission: An idea whose time has come," IEEE Communications magazine, vol. 28, 1990, p. 5-14.

[7] T.D. Chiueh et P. Tsai, OFDM Baseband Receiver Design for Wireless Communications, John Wiley \& Sons (Asia) Pte Ltd, 2007.

[8] T. Pollet, P. Spruyt, et M. Moeneclaey, "The BER performance of OFDM systems using non-synchronized sampling," Global Telecommunications Conference, 1994. GLOBECOM '94. Communications: The Global Bridge., IEEE, 1994, p. 257 vol.1, 253.

[9] M. Morelli, C. Kuo, et M. Pun, "Synchronization Techniques for Orthogonal Frequency Division Multiple Access (OFDMA): A Tutorial Review," Proceedings of the IEEE, vol. 95, 2007, p. 1427, 1394.

[10] "Digital Video Broadcasting (DVB); Framing structure, channel coding and modulation for digital terrestrial television," Juin. 2008.

[11] J. van de Beek, M. Sandell, et P. Borjesson, "ML estimation of time and frequency offset in OFDM systems," Signal Processing, IEEE Transactions on [see also Acoustics, Speech, and Signal Processing, IEEE Transactions on], vol. 45, 1997, p. 1805, 1800.

[12] P. Thevenon. PhD Manuscript. "S-band air interfaces for navigation systems: a focus on OFDM signals". 2010.

[13] P. Thevenon, O. Julien, C. Macabiau, D. Serant, S. Corazza, M. Bousquet, L. Ries, et T. Grelier, "Pseudo-Range Measurements Using OFDM Channel Estimation," ION GNSS 2009, 2009

[14] S.F. Cotter et B.D. Rao, "Sparse channel estimation via matching pursuit with application to equalization," IEEE Transactions on Communications, vol. 50, 2002, p. 374-377.

[15] Ettus website: www.ettus.com

[16] Gnuradio website: www.gnuradio.org 